\title{
Klyachko's Theorem in Semi-finite von Neumann Algebras
}

\author{
By
}

\author{
Tetsuo HARADA*
}

\begin{abstract}
Let $\mathfrak{A}$ be a von Neumann algebra and $\overline{\mathfrak{A}}$ be the set of all $\tau$-measurable operators. For positive elements $A$ and $B$ in $\overline{\mathfrak{A}}$ we prove that

$$
\int_{K} \mu_{s}(A+B) d s \leq \int_{I} \mu_{s}(A) d s+\int_{J} \mu_{s}(B) d s,
$$

where $\mu(\cdot)$ denotes the generalized s-number and $I, J$, and $K$ are on an analogue of the Klyachko list.
\end{abstract}

\section{$\S 1$. Introduction}

Recently, Klyachko [11] has shown that the possible eigenvalues $\alpha, \beta, \gamma$ of Hermitian $n \times n$ matrices $A, B$, and $C=A+B$ are characterized by a certain list of inequalities togather with the trace equality $\sum \gamma_{i}=\sum \alpha_{i}+\sum \beta_{i}$. If we set $\alpha=\left(\alpha_{1}, \ldots, \alpha_{n}\right), \beta=\left(\beta_{1}, \ldots, \beta_{n}\right)$, and $\gamma=\left(\gamma_{1}, \ldots, \gamma_{n}\right)$ in decreasing order, then this list of inequalities is of the form

$$
\sum_{k \in K} \gamma_{k} \leq \sum_{i \in I} \alpha_{i}+\sum_{j \in J} \beta_{j}
$$

for certain subsets $I, J, K$ of the same cardinality $r$, which are on the Klyachko list, with $r<n$ (i.e. This list includes $3 n-3$ inequalities: $\alpha_{1} \geq \alpha_{2} \geq \cdots \geq \alpha_{n}$, $\left.\beta_{1} \geq \beta_{2} \geq \cdots \geq \beta_{n}, \gamma_{1} \geq \gamma_{2} \geq \cdots \geq \gamma_{n}.\right)$. We set $\lambda(I)=\left(i_{r}-r, \ldots, i_{2}-2, i_{1}-1\right)$

Communicated by H. Okamoto. Received June 1, 2006.

2000 Mathematics Subject Classification(s): Primary 47B15; Secondary 46L10, 47A05.

Key words: von Neumann algebra, singular number, spectral scale.

*9-16-201 Hakozaki 1-chome, Higashi-ku, Fukuoka 812-0053, Japan.

e-mail: tharada@math.kyushu-u.ac.jp

(c) 2007 Research Institute for Mathematical Sciences, Kyoto University. All rights reserved. 
for $I=\left\{i_{1}, \ldots, i_{r}\right\}$. Then $(I, J, K)$ is on the Klyachko list exactly when the Littlewood-Richardson coefficient $C_{\lambda(I) \lambda(J)}^{\lambda(K)}$ is positive (cf. [6], [7], [8]). From the solution of the saturation conjecture by Knutson-Tao [12], one can show that this list is exactly what was conjectured by Horn [10].

There is a long history to find necessary conditions. Arrange the eigenvalues of a Hermitian matrix $X$ in decreasing order: $\lambda(X)=\left(\lambda_{1}(X), \ldots, \lambda_{n}(X)\right)$. For example, in 1912 H. Weyl found

$$
\lambda_{i+j-1}(A+B) \leq \lambda_{i}(A)+\lambda_{j}(B) \text { whenever } i+j-1 \leq n .
$$

Some other inequalities were found in 1949 by Ky Fan:

$$
\sum_{k=1}^{r} \lambda_{k}(A+B) \leq \sum_{k=1}^{r} \lambda_{k}(A)+\sum_{k=1}^{r} \lambda_{k}(B) \text { for any } r<n .
$$

It is easy to check $(\{i\},\{j\},\{i+j-1\})$ and $(\{1, \ldots, r\},\{1, \ldots, r\},\{1, \ldots, r\})$ are on the Klyachko list.

There are some generalizations for the above inequality (1). Friedland [4] proved the inequality (1) for positive compact operators. Bercovici-Li [2] proved the inequality (1) for self-adjoint operators in $I I_{1}$ factors. Their methods are simple and useful, but we think that the same method cannot apply for the continuous infinite case, since they used discrete and finite properties respectively. In this paper, we will prove the inequality (1) by another method for any positive $\tau$-measurable operators. Of course, this result includes the infinite case (in particular, the continuous infinite case). And also, when $\mathfrak{A}$ is a finite von Neumann algebra, we can prove the inequality (1) not only for positive operators but also for bounded self-adjoint operators. More precisely, Theorem 3.1 shows

$$
\int_{J_{0}} \mu_{s}(A+B) d s \leq \int_{J_{1}} \mu_{s}(A) d s+\int_{J_{2}} \mu_{s}(B) d s
$$

for positive operators $A, B$. Let $C, D$ be bounded self-adjoint operators in $\mathfrak{A}$, there exists a positive number $x$ such that $C+x I \geq 0, D+x I \geq 0$. Therefore, we get

$$
\int_{J_{0}} \mu_{s}(C+D+2 x I) d s \leq \int_{J_{1}} \mu_{s}(C+x I) d s+\int_{J_{2}} \mu_{s}(D+x I) d s .
$$

Since $\mu_{s}(C+x I)=\lambda_{s}(C)+x$ and $m\left(J_{0}\right)=m\left(J_{1}\right)=m\left(J_{2}\right)<\infty$ (see Definition $2.4)$, the above inequality implies

$$
\int_{J_{0}} \lambda_{s}(C+D) d s \leq \int_{J_{1}} \lambda_{s}(C) d s+\int_{J_{2}} \lambda_{s}(D) d s
$$




\section{$\S 2 . \quad$ Notation}

In this section, we will collect some definitions and basic facts. Throughout the paper, let $\mathfrak{A}$ be a von Neumann algebra on a Hilbert space $\mathfrak{H}$ with a faithful normal semi-finite trace $\tau$. Let $\overline{\mathfrak{A}}$ denote the set of all $\tau$-measurable operators.

Definition 2.1 ( $\tau$-measurable operators). $\quad$ A densely-defined closed operator $A$ affiliated with $\mathfrak{A}$ is said to be $\tau$-measurable if for each $\varepsilon>0$ there exists a projection $E$ in $\mathfrak{A}$ such that $E(\mathfrak{H}) \subset \mathcal{D}(A)$ and $\tau(1-E) \leq \varepsilon$. (Here, $\mathcal{D}(A)$ denotes the domain of $A$.)

Let $A$ be a closed densely-defined operator affiliated with $\mathfrak{A}$. Let $|A|=$ $\int_{0}^{\infty} \lambda d e_{\lambda}(|A|)$ be the spectral decomposition. Then it is easy to check that $A$ is $\tau$-measurable if and only if $\tau\left(1-e_{\lambda}(|A|)\right)<\infty$ for $\lambda$ large enough (cf. [3], [14]).

Definition 2.2 (The generalized $s$-number, The spectral scale). For a self-adjoint element $A \in \overline{\mathfrak{A}}$, the distribution function $d_{s}(A)$ is defined by

$$
d_{s}(A)=\tau\left(e_{(s, \infty)}(A)\right)
$$

where $e_{(s, \infty)}(A)$ is the spectral projection of $A$ corresponding to the interval $(s, \infty)$.

The generalized s-number is defined by

$$
\mu_{t}(A)=\inf \left\{s: d_{s}(|A|) \leq t\right\} \quad(0<t<\infty)
$$

while the spectral scale is defined by

$$
\lambda_{t}(A)=\inf \left\{s: d_{s}(A) \leq t\right\} \quad(0<t<\infty) .
$$

The above definitions correspond to the decreasing rearrangement of the eigenvalues of $|A|$, and $A$ respectively. (When $e_{[0, \infty)}(A)=\infty, \lambda_{t}(A)$ does not necessarily correspond to the decreasing rearrangement of the eigenvalues of $A$. Therefore, we use the spectral scale only for elements in a finite von-Neumann algebra.) Of course, for positive operators, both definitions are the same.

If $A$ is a $\tau$-measurable operator, then we have $d_{s}(|A|)<\infty$ for $s$ large enough and $\lim _{s \rightarrow \infty} d_{s}(|A|)=0$ as noted before. Moreover, $\mu_{t}(A), \lambda_{t}(A)$ are non-increasing and right continuous on $(0, \infty)$. See [3], [15] for detailed properties of the above functions.

Definition 2.3 (Continuous flag).

Let $\left\{e_{s}\right\}_{s \in[0, \infty)}$ be a net of projections in $\mathfrak{A}$ satisfying the following conditions. 
(i) $\tau\left(e_{s}\right) \leq s$

(ii) If $s \leq t$, then, $e_{s} \leq e_{t}$

Then, we call $\left\{e_{s}\right\}_{s \in[0, \infty)}$ a continuous flag on $\mathfrak{A}$.

Definition 2.4 (Admissibility).

Let $J$ be a measurable set in $[0, \tau(1))$. (Here, $m$ denotes Lebesgue measure.) For a continuous flag $\left\{e_{s}\right\}, \Omega_{J}\left(\left\{e_{s}\right\}, \tau\right)$ is the set of projections in $\mathfrak{A}$ defined by

$$
\begin{array}{r}
\Omega_{J}\left(\left\{e_{s}\right\}, \tau\right)=\left\{p \in \mathfrak{A}_{\text {proj. }} ; \tau(p)=m(J), \tau(1-p)=m\left(J^{c}\right), \tau\left(p \wedge e_{s}\right) \geq\right. \\
m\{J \cap[0, s]\}(\text { for all } s \in[0, \tau(1)))\} .
\end{array}
$$

Let $J_{0}, J_{1}, J_{2}$ be Lebesgue measurable sets in $[0, \tau(1))$ such that $m\left(J_{0}\right)=$ $m\left(J_{1}\right)=m\left(J_{2}\right)$ and $m\left(J_{0}^{c}\right)=m\left(J_{1}^{c}\right)=m\left(J_{2}^{c}\right)$. A triple $\left(J_{0}, J_{1}, J_{2}\right)$ is called admissible for continuous flags $\left(\left\{e_{s}\right\},\left\{f_{s}\right\},\left\{g_{s}\right\}\right)$, if the following condition holds:

$$
\Omega_{J_{0}}\left(\left\{e_{s}\right\}, \tau\right) \cap\left(\Omega_{J_{1}^{c}}\left(\left\{f_{s}\right\}, \tau\right)\right)^{\perp} \cap\left(\Omega_{J_{2}^{c}}\left(\left\{g_{s}\right\}, \tau\right)\right)^{\perp} \neq \emptyset,
$$

where $\left(\Omega_{J_{1}^{c}}\left(\left\{f_{s}\right\}, \tau\right)\right)^{\perp}=\left\{1-p ; p \in \Omega_{J_{1}^{c}}\left(\left\{f_{s}\right\}, \tau\right)\right\}$. Of course, when $\tau(1)<\infty$, the above requirements for the complement of sets are automatically met.

Definition 2.5. For $A \in \overline{\mathfrak{A}}$ we set

$$
\mathcal{S G}(A)=\inf _{\xi \in \mathfrak{H} \backslash\{0\}} \frac{\|A \xi\|}{\|\xi\|} .
$$

Proposition 2.1. Let $A, B$ be operators in $\overline{\mathfrak{A}}$.

1. $\|A \xi\| \geq \mathcal{S G}(A) \cdot\|\xi\|$ for $\xi \in \mathfrak{H}$.

2. $\mathcal{S G}(A B) \geq \mathcal{S G}(A) \cdot \mathcal{S G}(B)$.

3. $\mathcal{S G}(A)=\left\|A^{-1}\right\|^{-1}$, if $A$ is invertible.

Proof. 1. follows immediately from the definition of $\mathcal{S G}(\cdot)$.

2., 3. From the definition, we have

$$
\begin{aligned}
\mathcal{S G}(A B) & =\inf _{\xi \in \mathfrak{H} \backslash\{0\}} \frac{\|A B \xi\|}{\|\xi\|} \\
& \geq \inf _{\xi \in \mathfrak{H} \backslash\{0\}} \frac{\mathcal{S G}(A) \cdot\|B \xi\|}{\|\xi\|} \\
& =\mathcal{S G}(A) \cdot \mathcal{S G}(B),
\end{aligned}
$$




$$
\begin{aligned}
\mathcal{S G}(A) & =\inf _{\xi \in \mathfrak{H} \backslash\{0\}} \frac{\|A \xi\|}{\left\|A^{-1}(A \xi)\right\|} \\
& =\inf _{\xi \in \mathfrak{H} \backslash\{0\}}\left(\frac{\left\|A^{-1}(A \xi)\right\|}{\|A \xi\|}\right)^{-1} \\
& =\left(\sup _{\xi \in \mathfrak{H} \backslash\{0\}} \frac{\left\|A^{-1} \xi\right\|}{\|\xi\|}\right)^{-1} \quad(\text { by } A(\mathfrak{H})=\mathfrak{H}) .
\end{aligned}
$$

Definition 2.6. Let $A$ be an operator in $\overline{\mathfrak{A}}$ and $E$ be a projection in $\mathfrak{A}$. Then the restriction of $\mathcal{S G}(A)$ for a projection $E$ (denote it $\mathcal{S G}(A)_{E}$ ) is

$$
\mathcal{S G}(A)_{E}=\inf _{\xi \in E(\mathfrak{H}) \backslash\{0\}} \frac{\|A \xi\|}{\|\xi\|} .
$$

For this restriction $\mathcal{S G}(\cdot)_{E}$, an anologue of Proposition 2.1 is valid.

Proposition 2.2. Let $A, B$ be operators in $\overline{\mathfrak{A}}$ and $E, F$ be projections in $\mathfrak{A}$.

1. $\|A \xi\| \geq \mathcal{S G}(A)_{E} \cdot\|\xi\|$ for $\xi \in E(\mathfrak{H})$.

2. $\mathcal{S G}(A B)_{E} \geq \mathcal{S G}(A)_{E} \cdot \mathcal{S G}(B)_{E}$.

3. If $E(\mathfrak{H})$ is an invariant subspace of $A$ and $A E$ is invertible as an element in $B(E(\mathfrak{H}))$. then $\mathcal{S G}(A)_{E}=\left\|(E A E)^{-1}\right\|_{E}^{-1}$. Here, $\|\cdot\|_{E}$ is the usual operator norm in $B(E(\mathfrak{H}))$.

4. $\mathcal{S G}(E)_{E}=1$, if $E \neq 0$.

5. $\mathcal{S G}(A)_{E} \geq \mathcal{S G}(A)_{F}$, if $E \leq F$.

Proof. 1., 2. are trivial (as in the proof of Proposition 2.1). 
3. We compute

$$
\begin{aligned}
\mathcal{S G}(A)_{E} & =\inf _{\xi \in E(\mathfrak{H}) \backslash\{0\}} \frac{\|A \xi\|}{\|\xi\|} \\
& =\inf _{\xi \in E(\mathfrak{H}) \backslash\{0\}} \frac{\|E A E \xi\|}{\|\xi\|} \\
& =\inf _{\xi \in E(\mathfrak{H}) \backslash\{0\}} \frac{\|E A E \xi\|}{\left\|(E A E)^{-1} E A E \xi\right\|} \\
& =\left(\sup _{\xi \in E(\mathfrak{H}) \backslash\{0\}} \frac{\left\|(E A E)^{-1} E A E \xi\right\|}{\|E A E \xi\|}\right)^{-1} \\
& =\left(\sup _{\xi \in E(\mathfrak{H}) \backslash\{0\}} \frac{\left\|(E A E)^{-1} \xi\right\|}{\|\xi\|}\right)^{-1} \\
& =\left\|(E A E)^{-1}\right\|_{E}^{-1} .
\end{aligned}
$$

4. $\mathcal{S G}(E)_{E}=\inf _{\xi \in E(\mathfrak{H}) \backslash\{0\}} \frac{\|E \xi\|}{\|\xi\|}=1$.

5 .

$$
\begin{aligned}
\mathcal{S G}(A)_{E} & =\inf _{\xi \in E(\mathfrak{H}) \backslash\{0\}} \frac{\|A \xi\|}{\|\xi\|} \\
& \geq \inf _{\xi \in F(\mathfrak{H}) \backslash\{0\}} \frac{\|A \xi\|}{\|\xi\|} \\
& =\mathcal{S G}(A)_{F} .
\end{aligned}
$$

\section{$\S 3 . \quad$ Main Theorem}

Proposition 3.1. For $A \in \overline{\mathfrak{A}}$ we have

$$
\begin{aligned}
\mu_{t}(A) & =\inf \{\|A E\| ; E \text { is a projection in } \mathfrak{A} \text { with } \tau(1-E) \leq t\} \\
& =\sup \left\{\mathcal{S G}(A)_{E} ; E \text { is a projection in } \mathfrak{A} \text { with } \tau(E) \geq t\right\}
\end{aligned}
$$

Proof. The first equality is shown in [3]. We show the second equality. Let us denote the supremum in this proposition by $\alpha$. If $\alpha=0$, then we have $\mu_{t}(A) \geq \alpha$. So we can assume $\alpha>0$. Then, for a sufficiently small $\varepsilon>0$, there exists a projection $E$ with $\tau(E) \geq t$ such that

$$
0<\alpha-\varepsilon<\mathcal{S G}(A)_{E} .
$$


If $\xi \in E(\mathfrak{H}) \cap e_{[0, \alpha-\varepsilon]}(|A|)(\mathfrak{H}),\|\xi\|=1$, then we have

$$
\begin{gathered}
\left(A^{*} A \xi \mid \xi\right)=\|A \xi\|^{2} \geq \mathcal{S G}(A)_{E}^{2}>(\alpha-\varepsilon)^{2}, \\
\left(A^{*} A \xi \mid \xi\right)=\left\||A| e_{[0, \alpha-\varepsilon]}(|A|) \xi\right\|^{2} \leq(\alpha-\varepsilon)^{2} .
\end{gathered}
$$

Therefore $E \wedge e_{[0, \alpha-\varepsilon]}(|A|)=0$, and we compute

$$
\begin{aligned}
E & =E-E \wedge e_{[0, \alpha-\varepsilon]}(|A|) \\
& \sim E \vee e_{[0, \alpha-\varepsilon]}(|A|)-e_{[0, \alpha-\varepsilon]}(|A|) \\
& \leq 1-e_{[0, \alpha-\varepsilon]}(|A|) \\
& =e_{(\alpha-\varepsilon, \infty)}(|A|) .
\end{aligned}
$$

We thus get

$$
\tau\left(e_{(\alpha-\varepsilon, \infty)}(|A|)\right) \geq \tau(E) \geq t, \alpha-\varepsilon \leq \mu_{t}(A) .
$$

On the other hand, set $E=e_{\left[\mu_{t}(A), \infty\right)}(|A|)$. If $\mu_{t}(A)=0$, then we have $\mu_{t}(A) \leq \alpha$. So we can assume $\mu_{t}(A)>0$. As $\tau(E) \geq t$ and $\mathcal{S G}(A)_{E} \geq \mathcal{S G}(|A|)_{E}$ (Let $A=u|A|$ be the polar decomposition. Since $E(\mathfrak{H}) \subset \overline{|A|(\mathfrak{H})}$, we have $\mathcal{S G}(u)_{E}=1$.), we get

$$
\begin{aligned}
\alpha & \geq \mathcal{S G}(|A|)_{E} \\
& =\left\|(E|A| E)^{-1}\right\|_{E}^{-1} \\
& =\left\|\int_{\left[\mu_{t}(A), \infty\right)} \lambda^{-1} d e_{\lambda}(|A|)\right\|_{E}^{-1} \\
& \geq \mu_{t}(A) .
\end{aligned}
$$

Proposition 3.2. $\quad$ Let $A$ be a positive operator in $\overline{\mathfrak{A}}$. Let $J$ be a measurable set in $[0, \tau(1))$, and $p$ be a projection in $\mathfrak{A}$.

1. If $\tau\left(p \wedge e_{\left(\mu_{s}(A), \infty\right)}(A)\right) \geq m\{J \cap[0, s]\}$, then $\mu_{m\{J \cap[0, s]\}}(p A p) \geq \mu_{s}(A)$.

2. If $\tau\left(\mathcal{R}\left(p e_{\left(\mu_{s}(A), \infty\right)}(A)\right)\right) \leq m\{J \cap[0, s]\}$, then $\mu_{m\{J \cap[0, s]\}}(p A p) \leq \mu_{s}(A)$. Here, $\mathcal{R}\left(\right.$ pe $\left._{\left(\mu_{s}(A), \infty\right)}(A)\right)$ denotes the range projection of $\operatorname{pe}_{\left(\mu_{s}(A), \infty\right)}(A)$.

Proof. 1. If $\mu_{s}(A)=0$, then it is trivial. So we can assume $\mu_{s}(A)>0$. 
From Proposition 2.2, we compute

$$
\begin{aligned}
\mu_{m\{J \cap[0, s]\}}(p A p) & \geq \mathcal{S G}(p A p)_{p \wedge e_{\left(\mu_{s}(A), \infty\right)}(A)} \\
& \geq \mathcal{S G}(p)_{p \wedge e_{\left(\mu_{s}(A), \infty\right)}(A)} \cdot \mathcal{S G}(A)_{p \wedge e_{\left(\mu_{s}(A), \infty\right)}(A)} \\
& \geq \mathcal{S G}(A)_{e_{\left(\mu_{s}(A), \infty\right)}(A)} \\
& =\left\|\int_{\left(\mu_{s}(A), \infty\right)} \lambda^{-1} d e_{\lambda}(A)\right\|_{e_{\left(\mu_{s}(A), \infty\right)}(A)}^{-1} \\
& \geq \mu_{s}(A) .
\end{aligned}
$$

2. Consider the set of $\tau$-measurable operators $\overline{p \mathfrak{A} p}$. Since $p A p$ is in $\overline{\mathfrak{A}}$,

$\mu_{t}(p A p)=\inf \{\|p A p E\| ; E$ is a projection in $p \mathfrak{A} p$ with $\tau(p-E) \leq t\}$.

Therefore,

$$
\begin{aligned}
\mu_{m\{J \cap[0, s]\}}(p A p) & \leq\left\|p A p\left(p-\mathcal{R}\left(p e_{\left(\mu_{s}(A), \infty\right)}(A)\right)\right)\right\| \\
& =\left\|p A p\left(p \wedge e_{\left[0, \mu_{s}(A)\right]}(A)\right)\right\| \\
& =\left\|p A\left(p \wedge e_{\left[0, \mu_{s}(A)\right]}(A)\right)\right\| \\
& \leq\left\|A\left(p \wedge e_{\left[0, \mu_{s}(A)\right]}(A)\right)\right\| \\
& =\left\|A e_{\left[0, \mu_{s}(A)\right]}(A)\left(p \wedge e_{\left[0, \mu_{s}(A)\right]}(A)\right)\right\| \\
& \leq\left\|A e_{\left[0, \mu_{s}(A)\right]}(A)\right\| \\
& \leq \mu_{s}(A) .
\end{aligned}
$$

Proposition 3.3. $\quad$ Let $A$ be a positive operator in $\overline{\mathfrak{A}}$ and $J$ be a Lebesgue measurable set in $[0, \tau(1))$. Let $p$ be a projection in $\mathfrak{A}$ with $m(J)=\tau(p)$. Then,

$$
\int_{J} \mu_{m\{J \cap[0, s]\}}(p A p) d s=\int_{0}^{\tau(p)} \mu_{s}(p A p) d s=\tau(p A p) .
$$

Proof. It suffices to prove that $\mu_{m\{J \cap[0, s]\}}(p A p)$ and $\mu_{s}(p A p)$ have the same distribution function. At first we claim that

$$
m\left\{s \in J ; \mu_{m\{J \cap[0, s]\}}(p A p)>t\right\}=m\{J \cap[0, \alpha]\}(t>0)
$$

with $\alpha=\sup \left\{s \in J ; \mu_{m\{J \cap[0, s]\}}(p A p)>t\right\}$. 
Let $u$ be an element in $J$ such that $\mu_{m\{J \cap[0, u]\}}(p A p)>t$, then

$$
m\left\{s \in J ; \mu_{m\{J \cap[0, s]\}}(p A p)>t\right\} \geq m\{J \cap[0, u]\} .
$$

(since $\mu_{t}$ is decreasing for $s \leq u, \mu_{m\{J \cap[0, s]\}}(p A p)>t$ )

From the definition of $\alpha$ there exists $\left\{\alpha_{j}\right\}$ in $\left\{s \in J ; \mu_{m\{J \cap[0, s]\}}(p A p)>t\right\}$ such that $\alpha_{j} \nearrow \alpha$.

Therefore, we have $m\left\{s \in J ; \mu_{m\{J \cap[0, s]\}}(p A p)>t\right\} \geq m\left\{J \cap\left[0, \alpha_{j}\right]\right\}$ (for all $j$ ). Taking the $\lim _{j \rightarrow \infty}$ of the both sides, we get

$$
m\left\{s \in J ; \mu_{m\{J \cap[0, s]\}}(p A p)>t\right\} \geq m\{J \cap[0, \alpha]\} .
$$

Conversely, from the definition of $\alpha$, we obviously have

$$
m\left\{s \in J ; \mu_{m\{J \cap[0, s]\}}(p A p)>t\right\} \leq m\{J \cap[0, \alpha]\} .
$$

Finally, we will prove that $m\{J \cap[0, \alpha]\}=m\left\{s ; \mu_{s}(p A p)>t\right\}$. Clearly,

$$
\begin{gathered}
m\left\{J \cap\left[0, \alpha_{j}\right]\right\} \leq m\left\{s ; \mu_{s}(p A p)>t\right\} . \\
\left(\text { since } \mu_{m\left\{J \cap\left[0, \alpha_{j}\right]\right\}}(p A p)>t\right)
\end{gathered}
$$

So we get

$$
m\{J \cap[0, \alpha]\} \leq m\left\{s ; \mu_{s}(p A p)>t\right\} .
$$

On the other hand, we set

$$
\beta=\sup \left\{s ; \mu_{s}(p A p)>t\right\} .
$$

Since $\mu_{s}$ is decreasing, $m\left\{s ; \mu_{s}(p A p)>t\right\}=\beta$

If $\beta=\infty$, then $\alpha=\tau(p)=m(J)=\infty$. Therefore, we get the conclusion. So we can assume $\beta<\infty$. When we set

$$
f(x)=m\{J \cap[0, x]\} \quad(x \in[0, \tau(1))),
$$

$f$ is a measurable function on $[0, \tau(1))$. Futhermore, $f$ is a continuous finction on $[0, \tau(1))$.

Indeed, if $x_{j} \rightarrow x$, then

$$
\begin{aligned}
\lim _{j \rightarrow \infty} m\left\{J \cap\left[0, x_{j}\right]\right\} & =\lim _{j \rightarrow \infty} \int_{0}^{\tau(1)} \chi_{J \cap\left[0, x_{j}\right]} d m \\
& =\int_{0}^{\tau(1)} \lim _{j \rightarrow \infty} \chi_{J \cap\left[0, x_{j}\right]} d m
\end{aligned}
$$

(by the dominated convergence theorem)

$$
=m\{J \cap[0, x]\} \text {. }
$$


Since $f(0)=0, f(\tau(1))=m(J)=\tau(p), f$ attains any value in $[0, \tau(p)]$. Therefore, for all $\varepsilon>0$, there exists $x$ such that

$$
m\{J \cap[0, x]\}=\beta-\varepsilon .
$$

When we set $y=\sup \{J \cap[0, x]\}$,

$$
\{J \cap[0, x]\}=\{J \cap[0, y]\} .
$$

From the definition of $y$, there exists $\left\{y_{j}\right\}$ in $\{J \cap[0, x]\}$ such that $y_{j} \nearrow y$. We get

$$
\lim _{j \rightarrow \infty} m\left\{J \cap\left[0, y_{j}\right]\right\}=m\{J \cap[0, x]\}=\beta-\varepsilon .
$$

As $m\left\{J \cap\left[0, y_{j}\right]\right\} \leq \beta-\varepsilon$, we have $\mu_{m\left\{J \cap\left[0, y_{j}\right]\right\}}(p A p)>t$. Therefore we get $m\{J \cap[0, \alpha]\} \geq \lim _{j \rightarrow \infty} m\left\{J \cap\left[0, y_{j}\right]\right\}=\beta-\varepsilon \quad$ (from the definition of $\alpha, y_{j} \leq \alpha$ )

The proof is complete since $\varepsilon$ is arbitrary.

Proposition 3.4. $\quad$ Let $A$ be a positive operator in $\overline{\mathfrak{A}}$. Let $J$ be a measurable set, and $p$ be a projection in $\mathfrak{A}$.

1. If $p \in \Omega_{J}\left(\left\{e_{\left(\mu_{s}(A), \infty\right)}(A)\right\}, \tau\right)$, then $\tau(p A p) \geq \int_{J} \mu_{s}(A) d s$.

2. If $p \in\left(\Omega_{J^{c}}\left(\left\{e_{\left(\mu_{s}(A), \infty\right)}(A)\right\}, \tau\right)\right)^{\perp}$, then $\tau(p A p) \leq \int_{J} \mu_{s}(A) d s$.

Proof. 1. Proposotions 3.2 and 3.3 show the result.

2. From the Definition of $\left(\Omega_{J^{c}}\left(\left\{e_{\left(\mu_{s}(A), \infty\right)}(A)\right\}, \tau\right)\right)^{\perp}$,

$$
\tau\left((1-p) \wedge e_{\left(\mu_{s}(A), \infty\right)}(A)\right) \geq m\left\{J^{c} \cap[0, s]\right\} \quad(\text { for all } s \in[0, \tau(1))) .
$$

By substracting the both sides from $s$, we have

$$
s-\tau\left((1-p) \wedge e_{\left(\mu_{s}(A), \infty\right)}(A)\right) \leq s-m\left\{J^{c} \cap[0, s]\right\} \quad(\text { for all } s \in[0, \tau(1))) .
$$

We note

$$
\begin{aligned}
& s-\tau\left((1-p) \wedge e_{\left(\mu_{s}(A), \infty\right)}(A)\right) \\
& \geq \tau\left(e_{\left(\mu_{s}(A), \infty\right)}(A)\right)-\tau\left((1-p) \wedge e_{\left(\mu_{s}(A), \infty\right)}(A)\right) \\
& =\tau\left(e_{\left(\mu_{s}(A), \infty\right)}(A)-(1-p) \wedge e_{\left(\mu_{s}(A), \infty\right)}(A)\right) \\
& =\tau\left((1-p) \vee e_{\left(\mu_{s}(A), \infty\right)}(A)-(1-p)\right) \\
& =\tau\left(\mathcal{R}\left(p e_{\left(\mu_{s}(A), \infty\right)}(A)\right)\right),
\end{aligned}
$$


and

$$
\begin{aligned}
& s-m\left\{J^{c} \cap[0, s]\right\} \\
& =m\{[0, s]\}-m\left\{J^{c} \cap[0, s]\right\} \\
& =m\{J \cap[0, s]\} .
\end{aligned}
$$

Using Propositions 3.2 and 3.3, we get

$$
\tau(p A p) \leq \int_{J} \mu_{s}(A) d s
$$

Theorem 3.1. $\quad$ Let $A$ and $B$ be positive operators in $\overline{\mathfrak{A}}$. Let $J_{0}, J_{1}$ and $J_{2}$ be measurable sets with $m\left(J_{0}\right)=m\left(J_{1}\right)=m\left(J_{2}\right)$ and $m\left(J_{0}^{c}\right)=m\left(J_{1}^{c}\right)=$ $m\left(J_{2}^{c}\right)$. If a triple $\left(J_{0}, J_{1}, J_{2}\right)$ is admissible for $\left(\left\{e_{\left(\mu_{s}(A+B), \infty\right)}(A+B)\right\}\right.$, $\left.\left\{e_{\left(\mu_{s}(A), \infty\right)}(A)\right\},\left\{e_{\left(\mu_{s}(B), \infty\right)}(B)\right\}\right)$, then

$$
\int_{J_{0}} \mu_{s}(A+B) d s \leq \int_{J_{1}} \mu_{s}(A) d s+\int_{J_{2}} \mu_{s}(B) d s .
$$

Proof. If $p \in \Omega_{J_{0}}\left(\left\{e_{\left(\mu_{s}(A+B), \infty\right)}(A+B)\right\}, \tau\right) \cap\left(\Omega_{J_{1}^{c}}\left(\left\{e_{\left(\mu_{s}(A), \infty\right)}(A)\right\}, \tau\right)\right)^{\perp}$ $\cap\left(\Omega_{J_{2}^{c}}\left(\left\{e_{\left(\mu_{s}(B), \infty\right)}(B)\right\}, \tau\right)\right)^{\perp}$, then

$$
\tau(p(A+B) p) \geq \int_{J_{0}} \mu_{s}(A+B) d s \text { (by Proposition 3.4 ). }
$$

On the other hand, since $p \in\left(\Omega_{J_{1}^{c}}\left(\left\{e_{\left(\mu_{s}(A), \infty\right)}(A)\right\}, \tau\right)\right)^{\perp}$, Proposition 3.4 shows

$$
\tau(p A p) \leq \int_{J_{1}} \mu_{s}(A) d s .
$$

Similarly, for an operator $B$ we get

$$
\tau(p A p) \leq \int_{J_{2}} \mu_{s}(B) d s .
$$

Therefore, we obtain

$$
\begin{aligned}
\int_{J_{0}} \mu_{s}(A+B) d s & \leq \tau(p(A+B)) \\
& =\tau(p A)+\tau(p B) \\
& \leq \int_{J_{1}} \mu_{s}(A) d s+\int_{J_{2}} \mu_{s}(B) d s .
\end{aligned}
$$


Lemma 3.1. For $J=[0, r]$ we have

$$
\Omega_{J^{c}}\left(\left\{e_{\left(\mu_{s}(A), \infty\right)}(A)\right\}, \tau\right)^{\perp}=\left\{p \in \mathfrak{A}_{\text {proj. }} ; \tau(p)=r\right\} .
$$

Proof. Let $p$ be a projection with $\tau(p)=r$. It is enough to prove that

$$
\tau\left((1-p) \wedge e_{\left(\mu_{s}(A), \infty\right)}(A)\right) \geq m\left\{[0, s] \cap[0, r]^{c}\right\} .
$$

If $s \leq r$, it is trivial. When $r<s<\infty$, we get

$$
\begin{aligned}
\tau\left(\mathcal{R}\left(e_{\left(\mu_{s}(A), \infty\right)}(A) p\right)\right) & =\tau\left(\mathcal{R}\left(p e_{\left(\mu_{s}(A), \infty\right)}(A)\right)\right) \\
& \leq \tau(p) \\
& =r \\
& =m\{[0, s] \cap[0, r]\} .
\end{aligned}
$$

By substracting the both sides from $s$, we get the conclusion.

From Theorem 3.4 and Lemma 3.1 we obtain

$$
\begin{gathered}
\Omega_{[0, r]}\left(\left\{e_{\left(\mu_{s}(A+B), \infty\right)}(A+B)\right\}, \tau\right) \cap\left(\Omega_{[0, r]^{c}}\left(\left\{e_{\left(\mu_{s}(A), \infty\right)}(A)\right\}, \tau\right)\right)^{\perp} \\
\cap\left(\Omega_{[0, r]^{c}}\left(\left\{e_{\left(\mu_{s}(B), \infty\right)}(B)\right\}, \tau\right)\right)^{\perp}=\Omega_{[0, r]}\left(\left\{e_{\left(\mu_{s}(A+B), \infty\right)}(A+B)\right\}, \tau\right) .
\end{gathered}
$$

When $\mathfrak{A}$ has no minimal projection, $\Omega_{[0, r]}\left(\left\{e_{\left(\mu_{s}(A+B), \infty\right)}(A+B)\right\}, \tau\right)$ is not an empty set, because $e_{\left(\mu_{r}(A+B), \infty\right)}(A+B)$ is inclueded. Since we can always embed $\mathfrak{A}$ into $\mathfrak{A} \otimes L_{([0,1] ; d t)}^{\infty}$, we get the inequality

$$
\int_{0}^{r} \mu_{s}(A+B) d s \leq \int_{0}^{r} \mu_{s}(A) d s+\int_{0}^{r} \mu_{s}(B) d s \quad(\text { for all } r \in[0, \tau(1))) .
$$

This inequalty was found by Ky Fan when $\mathfrak{A}=M_{n}(\mathbb{C})$. For a general von Neumann algebra with a faithful semifinite normal trace several proofs are known (cf. [3], [9]).

\section{References}

[1] R. Bhatia, Matrix analysis, Springer, New York, 1997.

[2] H. Bercovici and W. S. Li, Inequalities for eigenvalues of sums in a von Neumann algebra, in Recent advances in operator theory and related topics (Szeged, 1999), 113126, Birkhäuser, Basel.

[3] T. Fack and H. Kosaki, Generalized s-numbers of $\tau$-measurable operators, Pacific J. Math. 123 (1986), no. 2, 269-300.

[4] S. Friedland, Finite and infinite dimensional generalizations of Klyachko's theorem, Linear Algebra Appl. 319 (2000), no. 1-3, 3-22. 
[5] W. Fulton, Young tableaux, Cambridge Univ. Press, Cambridge, 1997.

[6] Eigenvalues of sums of Hermitian matrices (after A. Klyachko), Astérisque No. 252 (1998), Exp. No. 845, 5, 255-269.

[7] Eigenvalues of majorized Hermitian matrices and Littlewood-Richardson coefficients, Linear Algebra Appl. 319 (2000), no. 1-3, 23-36.

[8] W. Fulton, Eigenvalues, invariant factors, highest weights, and Schubert calculus, Bull. Amer. Math. Soc. (N.S.) 37 (2000), no. 3, 209-249, math. AG/9908012.

[9] F. Hiai and Y. Nakamura, Majorizations for generalized $s$-numbers in semifinite von Neumann algebras, Math. Z. 195 (1987), no. 1, 17-27.

[10] A. Horn, Eigenvalues of sums of Hermitian matrices, Pacific J. Math. 12 (1962), 225-241.

[11] A. A. Klyachko, Stable bundles, representation theory and Hermitian operators, Selecta Math. (N.S.) 4 (1998), no. 3, 419-445.

[12] A. Knutson and T. Tao, The honeycomb model of $\mathrm{GL}_{n}(\mathbb{C})$ tensor products. I. Proof of the saturation conjecture, J. Amer. Math. Soc. 12 (1999), no. 4, 1055-1090.

[13] C.-K. Li and R. Mathias, The Lidskii-Mirsky-Wielandt theorem-additive and multiplicative versions, Numer. Math. 81 (1999), no. 3, 377-413.

[14] E. Nelson, Notes on non-commutative integration, J. Functional Analysis 15 (1974), 103-116.

[15] D. Petz, Spectral scale of selfadjoint operators and trace inequalities, J. Math. Anal. Appl. 109 (1985), no. 1, 74-82.

[16] I. E. Segal, A non-commutative extension of abstract integration, Ann. of Math. (2) 57 (1953), 401-457.

[17] E. M. Stein and G. Weiss, Introduction to Fourier analysis on Euclidean spaces, Princeton Univ. Press, Princeton, N.J., 1971.

[18] M. Terp, $L^{p}$-spaces associated with von Neumann algebras, Copenhagen Univ., 1981. 